\title{
Total Pinealectomy by an Occipital Parasagittal Approach in Sheep
}

Robert J. Dempsey, M.D., ${ }^{*}$ Jonathan Hopkins, M.D., ${ }^{*}$ Eric L. Bittman, Ph.D., ${ }^{\dagger \dagger}$ and Glenn W. Kindt, M.D.*

A reliable method of total pinealectomy in sheep without a resulting significant neurological deficit has been developed and is described. The known effect of light on sheep gonadotropin levels helps validate these studies of its pineal gland. Special attention is given to delineation of the surgical anatomy of the gland and its relationship under magnification to the central draining veins of the brain. In this large mammal this relationship differs considerably from that of humans. The application of an intrahemispheric occipital exposure using magnification, as well as the preoperative administration of steroids and osmotic agents, helped to minimize the effects of retraction and resultant cerebral edema. We have thus been able to increase the rate of succeissful pinealectomy to $83 \%$, allowing the development of groups of animals suitable for studies of the neuroendocrine function of the pineal and pituitary glands.

Dempsey RJ, Hopkins J, Bittman EL, Kindt GW: Total pinealectomy by an occipital parasagittal approach in sheep. Surg Neurol 18:377-380, 1982

The surgical anatomy of the pineal region has been a subject of great interest to neurosurgeons since the early work of Walter Dandy [2]. Clinical experience and detailed studies of cadavers continue to contribute to our understanding of this region [13]. The lack of a good large animal model, however, has made chronic survivor experiments of the effects of pinealectomy difficult.

Neuroendocrinologists have found the pineal gland a unique challenge since 1917 when McCord and Allen [11] first proved that extracts of the gland have a biological action. Lerner and his associates [8] later isolated melatonin as a prospective hormone secreted in a cyclic nocturnal pattern from the gland. Studies of biological rhythms have since suggested that the pineal may mediate the effects of

From the *Section of Neurosurgery, Department of Surgery, †Michigan Society of Fellows, Reproductive Endocrinology Program, and the $¥ D$ epartments of Pathology and Physiology, The University of Michigan, Ann Arbor, MI.

Address reprint requests to Dr. Robert J. Dempsey, Section of Neurosurgery, University of Michigan Hospital, 1405 E. Ann St.. Ann Arbor, MI 48109.

Key words: pineal gland; melatonin; pinealectomy; sheep; neuroendocrinology; pineal tumor. day-length $\S$ on the neuroendocrine system $[5,10,17]$. To allow a systematic study of these effects, a model was developed for pinealectomy in the adult ewe, a long-lived mammal whose seasonal reproductive rhythm is known to be regulated by photoperiodism [6].

Pinealectomy has been described in many laboratory animals $[1,2,4,9]$, including sheep [14]. Unlike that done in small laboratory rodents, whose pineal glands are superficially located, the sheep pineal gland is shielded by the central draining veins of the brain (Fig. 1). Previous attempts to develop a pinealectomy procedure in the ewe have failed to take this venous system into consideration and have resulted in a high frequency of complications (>45\%), often precluding successful pinealectomy [14].

The surgical anatomy of this animal's pineal region presented a challenge because of some marked differences from the human anatomy. An adaptation of the midline occipital approach developed by Poppen for human beings, was utilized in this model [12]. This allowed more flexibility in the exposure necessary for total gland removal in the very confined cranium of the sheep. By delineating the deep venous anatomy of a midline occipital approach to the gland, and by introducing neurosurgical techniques such as microsurgery, complications were reduced to a level acceptable for the production of substantial numbers of pinealectomized animals $(17 \%)$. This allowed for extended studies over many seasons in photoperiodic animals large enough for safe repeated assessments of serum levels of melatonin [15] and the later infusion of the appropriate hormone after removal of the gland.

\section{Materials and Methods}

Mature Suffolk ewes were maintained at the University of Michigan Sheep Research Facility. Each animal was given no food for 24 hours prior to operation. Eighteen hours before operation, ewes were given intramuscular injections of ampicillin ( $5 \mathrm{mg}$ per kilogram of body weight) and dexamethasone $(0.6 \mathrm{mg} / \mathrm{kg})$. Immediately prior to anesthesia, Turcapsol" and Emulsifol* were administered orally as car-

$\S$ The ability of the neuroendocrine system to determine seasons of the year by measuring the length of day, thus allowing accurate seasonal reproductive patterns.

"Salicylic acid, methyl salicylate, camphor, oil of turpentine, and ginger; Pitman-Moore, Washington Crossing, NJ.

\#Soybean oil and polysorbate; Roberts Laboratories, Rockford, IL. 


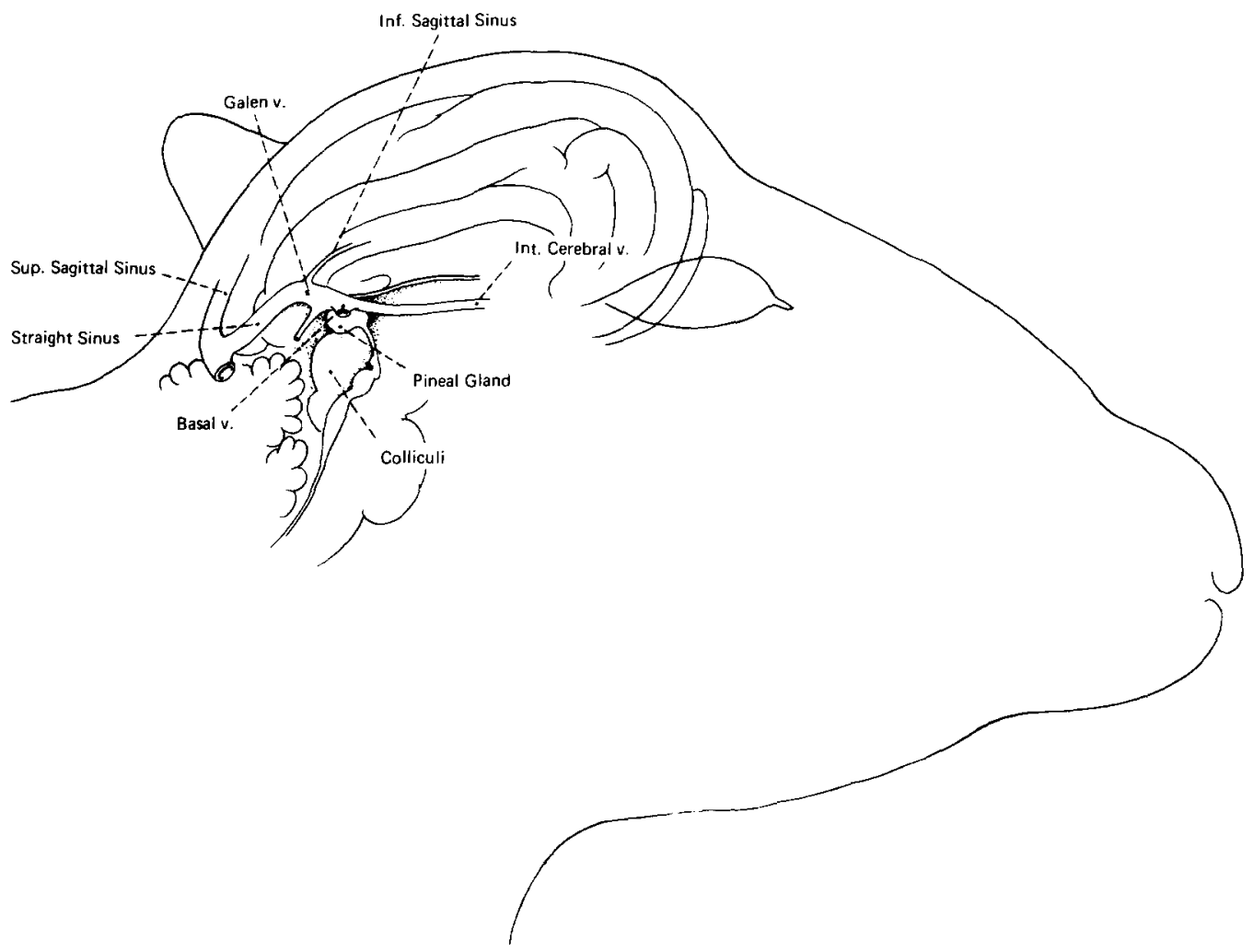

minative and antifermentative agents. Mannitol $(0.25$ $\mathrm{gm} / \mathrm{kg})$ and dexamethasone $(0.12 \mathrm{mg} / \mathrm{kg})$ were given intravenously at this time to facilitate retraction of the brain.

Anesthesia was induced with intrajugular sodium pentobarbital $(14 \mathrm{mg} / \mathrm{kg})$. Endotracheal methoxyflurane was used for the remainder of the operation. Animals were placed in a prone position, and the head was shaved, scrubbed, and draped to provide a sterile operative field extending from the horn buds posteriorly, approximately $10 \mathrm{~cm}$, to the base of both ears laterally.

\section{Surgical Procedure}

A left parietooccipital craniectomy was fashioned by making a U-shaped flap based laterally, and extending to cross and expose the midline and parietal skull anterior to the attachment of the aponeurosis of the neck musculature. Hemostasis was achieved by electrocautery and hemostats. A bit-and-brace perforator was used to create a left parietooccipital burrhole $1 \mathrm{~cm}$ lateral to the midline and 1 $\mathrm{cm}$ anterior to the aponeurosis. This was then enlarged to a $2.5 \times 2.5 \mathrm{~cm}$ craniectomy, using an angled rongeur. The craniectomy crossed the midline to expose the sagittal sinus, but needed to be no larger than this to avoid undue exposure of the cortex.

The dura mater was opened using scalpels and scissors, and reflected back medially to protect the sagittal sinus. This opening allowed exposure of the left parietooccipital cortex as it meets the midline of the falx cerebri. At this
Fig. 1. The sheep pineal gland. Note its central position and envelopment by the central draining veins of the brain.

point, a surgical loupe was used to provide magnification. The operating microscope may also be used, thereby increasing both illumination and magnification. Medial draining veins from the cortex to the sagittal sinus were identified, electrocoagulated with bipolar current, and cut. Only then was the occipital lobe gently retracted from the midline of the falx using a ribbon retractor. Prior use of mannitol protected the brain from the effects of retraction, allowing adequate exposure. A persistent vein draining the occipital lobe into the lower falx at the margin of the straight sinus was seen in all animals. This vein was coagulated prior to retraction to avoid hemorrhage. It is shown with a hemoclip in Figure 2.

At this point, lateral retraction of the occipital lobe revealed the tentorium cerebelli, which in the ewe is a natural extension of the falx. The tentorium runs parallel to the neck, unlike its more perpendicular relationship in humans. Owing to this relationship, however, the midbrain could be exposed anterior to the tentorium. It was not necessary to open the tentorium as in humans [12]. The quadrigeminal cistern was opened with a nerve hook, and the cerebrospinal fluid was drained to allow exposure of the midline of the collicular plate; this was well marked by the 


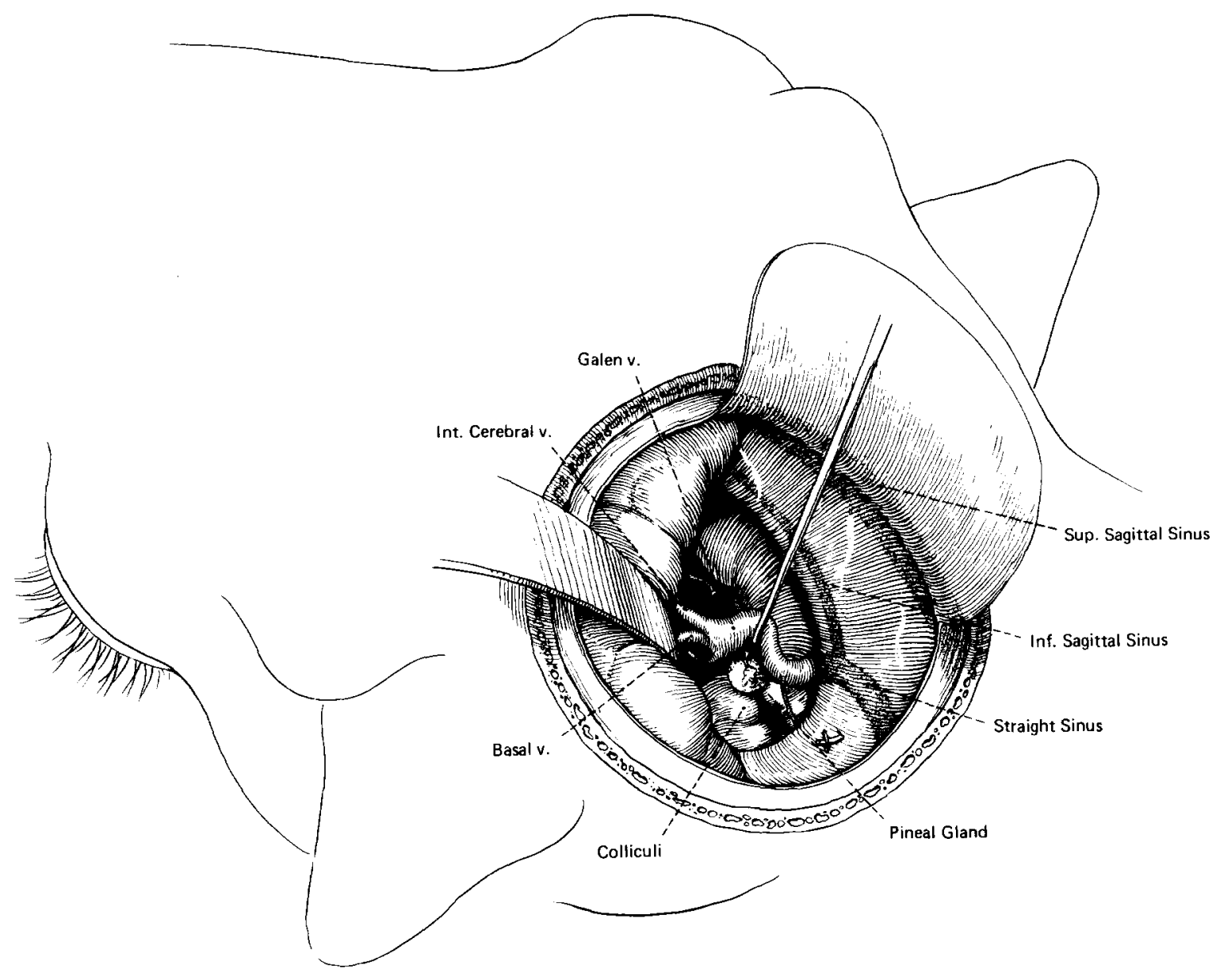

Fig. 2. The surgical approach, magnified. The gland is located inferior to the vein of Galen and superior to the quadrigeminal plate of the midbrain.

groove between the superior colliculi directly beneath the falx cerebri.

At this point, the pineal gland lies within the arachnoid anterior to the rostral colliculi. The gland is innervated by tiny postganglionic sympathetic branches that are of functional but not surgical concern. Its dissection, however, involved the preservation of the large venous structures that envelop it. The venous drainage of the pineal gland itself is accomplished by fine midline branches draining into the vein of Galen, which acts as a collection point for central ventricular and basilar branches. These small veins of the pineal gland were dissected free of the vein of Galen and a nerve hook was used to elevate the large vein (see Fig. 2). Careful dissection bilaterally freed the gland from the internal cerebral and basal veins. Although these may be adherent, they do not drain the gland and were bluntly freed from the pineal. The small midline precerebellar vein can be electrocauterized, if necessary, for removal of the gland. Once freed from its vascular pedicle, the pineal was grasped with a pituitary biopsy forceps, or other atraumatic forceps, and gently teased from beneath the vein of Galen. At this point hemostasis was achieved by Gelfoam and irrigation.

To accomplish closure, the retractor was removed, the dura mater was then loosely approximated, and the scalp was closed in a watertight fashion using absorbable suture material. (If the retraction and exposure had been kept small and no major structures or veins were damaged, edema was usually minimal.) The small size of the craniectomy made it unnecessary to replace any bone.

Completeness of pinealectomy was judged by inspection of the removed gland and serial determinations of serum melatonin levels. The normal fivefold nocturnal melatonin rise was successfully eliminated by pinealectomy.

\section{Results}

Twenty-seven ewes were pinealectomized in this fashion. The follow-up period ranged from 15 to 29 months. Initial attempts were complicated by an approximate $50 \%$ rate of hemorrhage or edema, as in earlier studies [14]. Once the anatomy of the ewe's deep cerebral vasculature was under- 
stood, the last 12 animals were operated on with a success rate of $83 \%$. The serial determination of serum melatonin levels in the pinealectomized animals showed that the normal nocturnal rise of serum melatonin to levels of 250 $\mathrm{pg} / \mathrm{ml}$, from daytime baselines of 25 to $45 \mathrm{pg} / \mathrm{ml}$, had been successfully eliminated. It is believed that our success was due to the application of neurosurgical techniques and the delineation of the pertinent surgical anatomy, thus allowing preservation of adjacent structures.

The procedure we used was a modification of the transtentorial approach to the posterior third ventricle used in humans, as described by Poppen [12]. Unlike Poppen, we have worked directly along the midline of the falx, with the administration of mannitol to allow gentle retraction and provide adequate exposure. This modification was attempted by Dandy in his experimental work, but he abandoned this approach after noting deaths in 12 dogs due to venous hemorrhage [3].

\section{Discussion}

The ewe's smaller brain and falx and relatively larger quadrigeminal plate make determination of the midline of the midbrain very straightforward. Because the angle formed by the head and neck in the ewe is more oblique than in humans, this causes the vital central venous structures of the ewe's brain to overlie the position of the pineal gland (see Fig. 1). Safe removal is dependent on dissecting the gland from beneath the vein of Galen into which its venous system drains. The adjacent paired internal cerebral and basal veins are then swept anteriorly off the gland, and it is removed through the angle formed by the vein of Galen and the ipsilateral basal vein (see Fig. 2).

The key anatomical difference between the ewe and the human is the parallel relationship of the tentorium to the neck, making infratentorial or transtentorial approaches to the area unrewarding. The infratentorial approach, as described by Krause [7] and later by Stein [16], has the advantage, in humans, of providing access to a midline tumor in the pineal region without obstruction by the deep venous system $[7,16]$. Rhoton [13] points out, however, that it is not well suited to a tumor that has considerable extension above the tentorium. A midline supratentorial approach, modifying Poppen's transtentorial approach, can provide wide exposure to the area. This is as useful in the animal model for circumferential dissection of venous structures from the gland as it would be in humans, should total removal of an unknown mass with supratentorial extension be contemplated. Laboratory experience with this model then allows the surgeon to develop experience with a rather versatile procedure, while demanding meticulous technique and stressing the identification and preservation of normal venous anatomy.

The sheep pinealectomy model, in addition to giving the surgeon experience in preserving the complex venous anatomy of the region, will also make possible the development of groups of pinealectomized ewes that have no other significant neurological deficit and that are suitable for large-scale neuroendocrine research. This availability allows the systematic study of such problems as the relationship of pineal dysfunction to such disorders as precocious puberty and panhypopituitarism seen in some tumors of the pineal region.

Supported by NIH grants HO-08333 to Dr. Fred J. Karsch, and HD05914 to Dr. Eric L. Bittman.

\section{References}

1. Braun RK, Gidean LA, Reibold TW, Peters RR: Surgical approach for pinealectomy in the calf. Am J Vet Res 38:1973-1976, 1977

2. Dandy WE: An operation for the removal of pineal tumors. Surg Gynecol Obstet 33:113-119, 1921

3. Dandy WE: Extirpation of the pineal body. J Exp Med 22:237-247, 1915

4. Hoffman RA, Reiter RJ: Rapid pinealectomy in hamsters and other small rodents. Anat Rec 153:19-22, 1965

5. Johnson LY, Reiter RJ: The pineal gland and its effects on mammalian reproduction, in Hubinont PO (ed): Progress in Reproductive Biology. Basel: Karger, 1969, Vol 4, pp 116-156

6. Karsch FJ: Seasonal reproduction: a saga of reversible fertility. Physiologist 23:29-38, 1980

7. Krause F: Operative Freilegung der Vierhügel, nebst Beobachtungen über Hirndruck und Dekompression. Zentralbl Chir 53:2812-2819, 1926

8. Lerner $\mathrm{AB}$, Case JO, Takahashi $\mathrm{Y}$, Lee $\mathrm{TH}$, Mori W: Isolation of melatonin, the pineal gland factor that lightens melanocytes. J Am Chem Soc 80:2587, 1958

9. Letellier MA, Plotka ED, Seal US, Verme LJ, Ozoga JJ: Technique for pinealectomy in deer, with notes on the neuroanatomy. Am J Vet Res 39:1617-1620, 1978

10. Lincoln GA, Short RV: Seasonal breeding: nature's contraceptive. Recent Prog Horm Res 36:1-43, 1980

11. McCord CP, Allen FP: Evidence associating pineal gland function with alteration in pigmentation. J Exp Zool 23:207-224, 1917

12. Poppen JL: The right occipital approach to a pinealoma. J Neurosurg 25:706-710, 1966

13. Rhoton AL, Yamamoto I, Peace DA: Microsurgery of the third ventricle. Part 2: Operative approaches. Neurosurgery 8:357-373, 1981

14. Roche JF, Dzuik PJ: A technique for pinealectomy of the ewe. Am J Vet Res 30:2031-2035, 1969

15. Rollag MD, Niswender GD: Radioimmunoassay of serum concentrations of melatonin in sheep exposed to different lighting regimes. Endocrinology 98:482-489, 1976

16. Stein MB: The infratentorial supracerebellar approach to pineal lesions. J Neurosurg 35:197-202, 1971

17. Wurtman RJ, Axelrod J, Kelly DE: The Pineal. New York, London: Academic Press, 1968, p 128 